\title{
Broadband Second Harmonic Parametric Scattering in Ferroelectric Crystals with Random Domains Structure
}

\author{
C. Cojocaru ${ }^{1}$, J. Trull ${ }^{1}$, R. Fischer ${ }^{2}$, S. M. Saltiel ${ }^{2,3}$, W. Krolikowski ${ }^{4}$, K. Staliunas $^{1}$, R. Herrero ${ }^{1}$ \\ R. Vilaseca ${ }^{1}$, Yuri S. Kivshar ${ }^{2}$ \\ 1) Departament de Física i Enginyeria Nuclear, Universitat Politècnica de Catalunya \\ Colom 11, 08222 Terrassa, Barcelona, Spain \\ 2) Centre for Ultrahigh Bandwidth Devices for Optical Systems (CUDOS), Nonlinear Physics Centre, Research \\ School of Physical Sciences and Engineering, Australian National University, Canberra, ACT 0200, Australia \\ 3) Faculty of Physics, University of Sofia, 5 J. Bourchier Boulevard, BG-1164, Sofia, Bulgaria \\ 4) Centre for Ultrahigh Bandwidth Devices for Optical Systems (CUDOS), Laser Physics Centre, Research \\ School of Physical Sciences and Engineering, Australian National University, Canberra, ACT 0200, Australia
}

\begin{abstract}
We report the results of an experimental study on broadband second-harmonic (SH) parametric scattering in an un-poled Strontium Barium Niobate (SBN) crystal with random-size ferroelectric domains. We consider different geometries of the interacting waves. The observed spatial distribution of the SHG is due to the larger facility to achieve phase matching conditions in the presence of disorder, and the polarization of the SH light can be both ordinary and extraordinary. The nonlinear coefficients of the crystal may be determined from the measurement of the SHG as a function of the fundamental beams polarization. The experimental results are in very good agreement with the predictions of our theoretical model.
\end{abstract}

Keywords: nonlinear optics; harmonic generation, random media.

\section{INTRODUCTION}

Nonlinear parametric processes in optics are known to depend critically on the phase matching between the parametrically interacting waves. Most frequently, phase matching is achieved by utilizing the crystal birefringence [1] or by employing the so-called quasi-phase-matching (QPM) technique [2]. The phase matching requirements enable efficient nonlinear interactions but they limit the spectral bandwidth of the nonlinear optical process, often making the designed frequency converter only suitable for a fixed input wavelength and single interaction. Recently it has been shown that disordered nonlinear media allow the phase matching in a broadbandwidth regime of the frequency conversion [3]-[5].

In this work, we experimentally study the coherence and polarization properties of the SH waves generated in an un-poled Strontium Barium Niobate (SBN) crystal, which is an example of such disordered nonlinear media. The SBN crystal is composed of micrometric random-size anti-parallel ferroelectric domains that allow to phasematch any second-order parametric process, including the SHG, over a broad range of wavelengths (limited only by the transparency window of the crystal, 0.4-6 $\mu \mathrm{m})$, without any poling.

\section{EXPERIMENTAL SET-UP AND RESULTS}

We study different types of SH generated signals using two different experimental configurations. In the first configuration we generate $\mathrm{SH}$ signal using two fundamental beams that make a small relative angle (quasicollinear second harmonic (QCSH) experiment). The fundamental beam at $1064 \mathrm{~nm}$ is provided by a Nd:YAG laser delivering $8 \mathrm{~ns}$ pulses. As can be seen in Fig.1 (a) the experimental set-up is design to deliver two beams (A and $\mathrm{B}$ ) that intersect at an external angle of $4^{\circ}$ within an unpoled SBN crystal. In the second configuration, the two fundamental beams are contra-propagating and the generated $\mathrm{SH}$ propagates transversally to the fundamental beam (transverse second harmonic (TSH) experiment). This experiment employs a Ti:Sapphire laser, tuneable in the range of $800-900 \mathrm{~nm}$, that delivers pulses of $120 \mathrm{fs}$. The set-up is arranged to deliver two counter-propagating pulses that meets in the central part of the SBN crystal. The use of the fs pulses is essential for this geometry as the pulse length is shorter that the crystal length, allowing to distinguish between the single beam contribution of the SH signal and SH due to the two beam mixing (see Fig.1 (b)).

In both experimental set-ups the polarization orientation of each entering beam is controlled by a $\lambda / 2$ plate. The $4 \mathrm{~mm}$ point symmetry group of the SBN crystal determines the nonzero components of the second-order susceptibility tensor, $\mathrm{d}^{(2)}$. Since the direction of the fundamental beams is close or coincides with the crystallographic $x$-axis, the relevant components are $d_{33}$ and $d_{32}=d_{24}$. 
a)

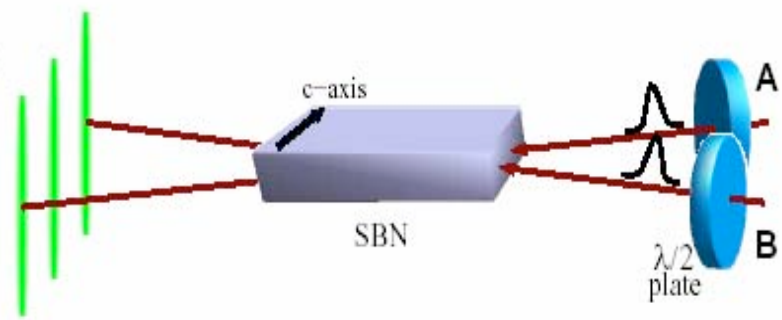

b)
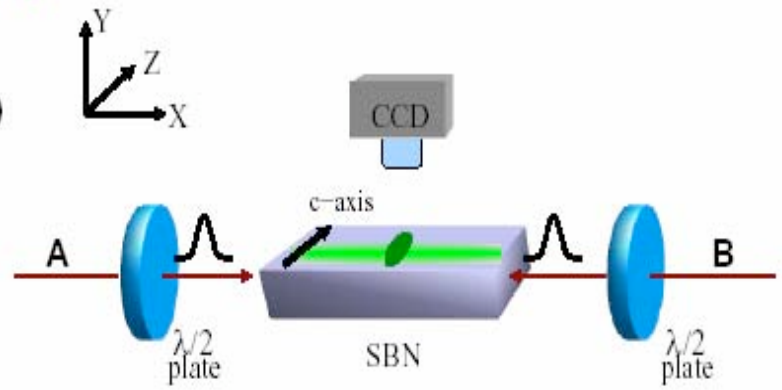

Figure 1. Schematic representation of the two experiments:

(a) QCSH experiment with the observation of a single SH beam and SH with small noncollinear angle of the fundamental beams;

(b) TSH experiment with a single pulse and/or two counter-propagating pulses. In both cases the optical c-axis of the crystal is perpendicular to the plane of drawing.

In Fig. 2(a) we show the photographs of the forward emitted SH signal observed with intersecting fundamental beams in the QCSH experiment. The three images (left to right) represent the case of (I) two extraordinary polarized fundamental beams, (II) perpendicularly polarized fundamental beams (A- extraordinary; B- ordinary), and (III) two ordinary polarized fundamental beams. The SH signal is emitted in the form of three well resolved vertical lines. The side lines (numbered 1, 3, 4, 6, 7, and 9) represent the SH signal emitted separately by each of the fundamental beams. The middle lines (numbered 2, 5, and 8) appear only when both the beams A and B are present simultaneously and hence represent the non-collinear SHG by two fundamental beams. The polarization state of each SH lines is marked as "e" (extraordinary) or "o" (ordinary). We notice that all but one outputs (line 5 ) are extraordinary polarized. The presence of this particular ordinary polarized signal defies the previous claims that in SBN crystals only extraordinary SH signal could be generated [6]. This line appears as a result of the parametric process $\mathrm{E}_{1 \mathrm{~A}} \mathrm{O}_{1 \mathrm{~B}}-\mathrm{O}_{2}$ that is governed by the same component $\mathrm{d}_{32}$ also responsible for the process $\mathrm{O}_{1 \mathrm{~A}} \mathrm{O}_{1 \mathrm{~B}}-\mathrm{E}_{2}$. However, since the phase mismatch for the former interaction is larger, the emitted $\mathrm{SH}$ signal is weaker than that in the latter process. We believe this experiment is the first one showing that such process is indeed possible in a SBN crystal.

The phase-matching conditions $\mathbf{k}_{1 \mathrm{~A}}+\mathbf{k}_{1 \mathrm{~B}}+\mathbf{g}=\mathbf{k}_{2}$, is represented in Fig 2 (b), where $\mathbf{k}_{1 \mathrm{~A}}$ and $\mathbf{k}_{1 \mathrm{~B}}$ represent the fundamental waves, $\mathbf{k}_{2}$ is for the $\mathrm{SH}$ wave, and $\mathbf{g}$ is one of the grating vectors supplied by a random structure of the nonlinear quadratic medium. The vectors $\mathbf{g}$ have different sizes and orientation, but they always lie in the xy-plane. Due to the randomness of the unpoled SBN crystal, a set of grating vectors with different magnitudes and orientations is available for phase matching. This enables phase matching in an extremely broad range of wavelengths and the $\mathrm{SH}$ emission in a broad angular range, as is seen in the images shown in Fig. 2(a). Moreover, as is demonstrated below, this continuum of grating vectors allows for simultaneous phase matching of several nonlinear parametric processes.

The SH signals recorded in the TSH experiment for the e-polarized counterpropagating beams are shown in Fig. 2(c). This choice of the input polarizations gives the largest intensity of the SH signal. In Fig. 2(c), the horizontal line is given by the SH process originating from each of the beams separately. If both the beams are present, the intensity of this line is just the sum of the intensities of two single-beam SHG process: AA-S and $\mathrm{BB}-\mathrm{S}$. The central bright peak is a result of the AB-S interaction in the position where two counter-propagating fs pulses overlap, and it represents the autocorrelation signal of the pulses [7]. In this geometry, the transversely emitted SH wave can only be extraordinary polarized (along the z-axis). 
(a)

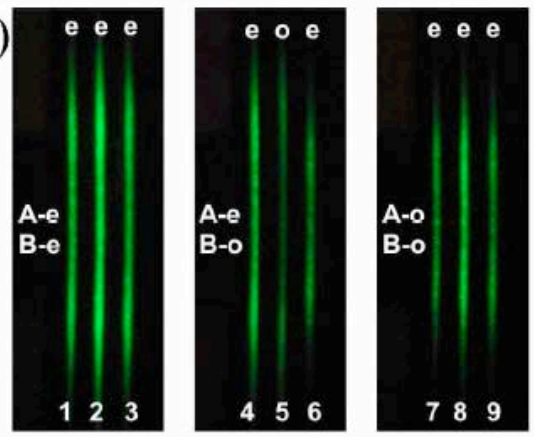

(b)

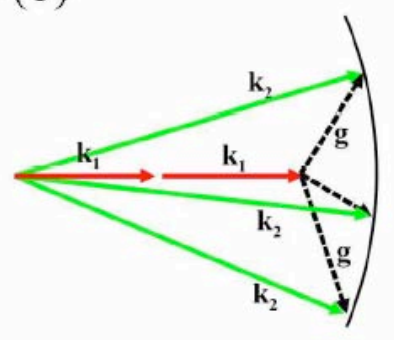

(c)

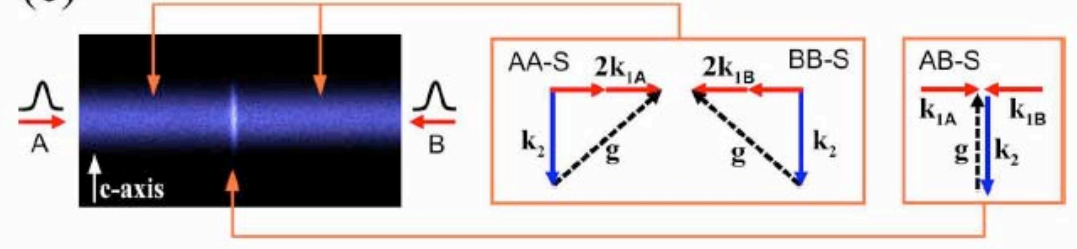

Figure 2. (a) SH output spatial distribution in a quasi-collinear geometry with two fundamental beams $A$ and $B$ of polarizations $o$ and $e$, lying in a horizontal plane and forming a small angle. The reciprocal-lattice vectors of the material structure lie in a vertical plane. (b) Examples of phase-matching geometries for such configuration. (c) Counterpropagating-pulse configuration; SHG generation occurs in transverse directions.

By varying, in both configurations, the polarization of the input fundamental beams, we demonstrate several different parametric processes including collinear, non-collinear and transverse second-harmonic generation that, in most cases, occur simultaneously contributing to the overall strength of the SH signal. The experimental measurement of the dependence of the SH intensity as a function of the input polarization of the fundamental beams in both experimental configurations are summarized in Fig. 3 (a-d)
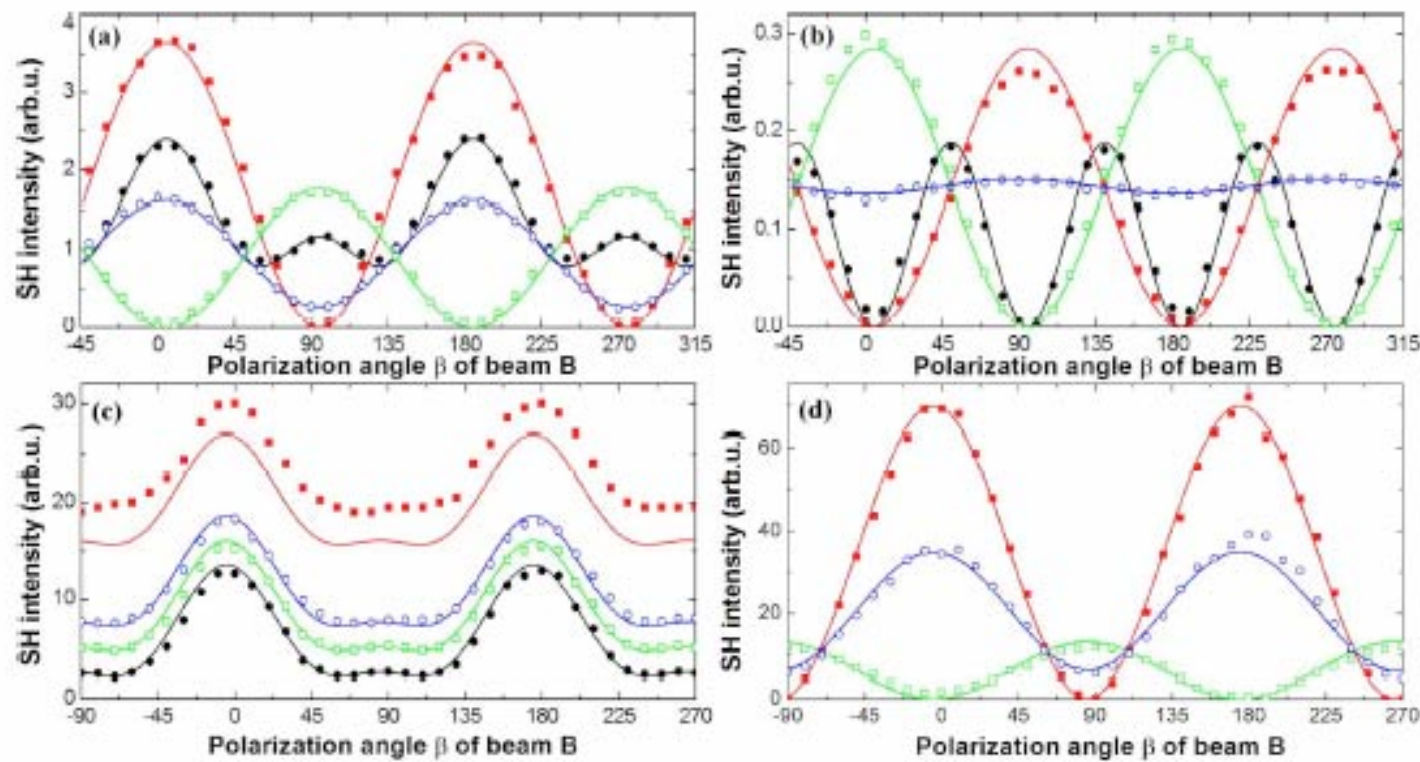

Figure 3. Experimentally observed SH signal vs. input polarization angle $\beta$ for three fixed polarizations of beam A: extraordinary - red full squares; mixed - blue open circles, and ordinary - green open squares. Single beam SH (black full circle); Lines show theoretical results. (a) extraordinary SH; (b) ordinary SH; both (a) and (b) are for SHG in the QCSH experiment. (c) Background TSH and single beam TSH; (d) TSH emission via A and B pulse mixing. Mixed polarizations: $\alpha=30^{\circ}, 43.5^{\circ}, 45^{\circ}, 45^{\circ}$ for $a, b, c$, $d$ plot respectively. 
In all this plots the polarization of the beam B is varied, while the polarization of the beam A is set to one of the following states: extraordinary, mixed or ordinary. The continuous lines shows the results of our theoretical results obtained when we consider that there is no interference between the contributions of different processes to the SH signal. We note a very good agreement between the experimental and theoretical results that confirms that disorder in the domain distribution in our crystal causes simultaneous processes to contribute incoherently into the overall SH signal. Unlike in a perfect periodic structures, where signals generated by different processes are coherent we prove that the disordered medium leads to an incoherent superposition of different processes that occur simultaneously.

We have employed the analysis of the polarization properties of the parametric processes to determine the relative strength of two relevant components of the second-order susceptibility tensor, such as the ratio $d_{32} / d_{33}$, and we have obtained a value of 0.44 (see for details [8]). Finally, we show that measuring the power of the second harmonic in a few different processes allows one to determine the statistical properties of the disordered domain distribution such as an average size of the domains $a=3.25 \mu \mathrm{m}$ and their dispersion $\sigma=1.15 \mu \mathrm{m}$. These values are consistent with the ones shown in the literature $[9,10]$.

\section{CONCLUSIONS}

We have studied the SHG in unpoled quadratic nonlinear crystals with disordered ferroelectric domains. We have demonstrated that such crystals enable the realization of several different parametric processes including collinear, non-collinear and transverse second-harmonic generation. In most of the cases, these parametric processes occur simultaneously, contributing incoherently to the overall strength of the second-harmonic signal. We have analyzed the polarization properties of the parametric processes and employed them to determine the relative strength of two relevant components of the second-order susceptibility tensor, such as the ratio $d_{32} / d_{33}$. We have demonstrated that, by measuring the power of the second harmonics in a few different processes, we can determine the statistical properties of the disordered domain distribution such as an average size of the domains and their dispersion.

\section{ACKNOWLEDGEMENTS}

The authors from Universitat Politècnica de Catalunya thank the project FIS2005-07931-C03-03. The Australian researchers acknowledge support by the Australian Research Council. Solomon Saltiel thanks the Australian National University and Universitat Politècnica de Catalunya for hospitality and support.

\section{REFERENCES}

[1] F. Zernike and J. E. Midwinter, Applied Nonlinear Optics (Wiley, New York, 1973).

[2] M. M. Fejer, G. A. Magel, D. H. Jundt, and R. L. Byer, "Quasi-phase-matched second harmonic generation: tuning and tolerances," IEEE J. Quantum Electron. QE-28, 2631-2654 (1992).

[3] M. Baudrier-Raybaut, R. Haidar, Ph. Kupecek, Ph. Lemasson, and E. Rosencher, "Random quasi-phasematching in bulk polycrystalline isotropic nonlinear materials," Nature (London) 432, 374-376 (2004).

[4] S. E. Skipetrov, "Disorder is the new order," Nature (London) 432, 285-286 (2004).

[5] R. Fischer, D. N. Neshev, S. M. Saltiel, W. Krolikowski, and Yu. S. Kivshar, "Broadband femtosecond frequency doubling in random media," Appl. Phys. Lett. 89, 191105(3) (2006).

[6] M. Horowitz, A. Bekker, and B. Fischer, "Broadband second-harmonic generation in SrBaNb2O6 by spread spectrum phase matching with controllable domain gratings," Appl. Phys. Lett. 62, 2619-2621 (1993).

[7] R. Fischer, D. N. Neshev, S. M. Saltiel, A. A. Sukhorukov, W. Krolikowski, Yu. S. Kivshar, "Monitoring ultrashort pulses by transverse frequency doubling of counterpropagating pulses in random media," Appl. Phys. Lett. 91, 031104(3) (2007).

[8] J. Trull, C. Cojocaru, R. Fischer, S. Saltiel, K. Staliunas, R. Herrero, R. Vilaseca, D. Neshev, W. Krolikowski, Y. Kivshar "Second-harmonic parametric scattering in ferroelectric crystals with disordered nonlinear domain structures", Optics Express 15, 15868 (2007).

[9] J. J. Romero, C. Arago, J. A. Gonzalo, D. Jaque, and J. Garcia Sole, "Spectral and thermal properties of quasiphase-matching second-harmonic generation in $\mathrm{Nd} 3+: \mathrm{Sr} 0.6 \mathrm{Ba} 0.4(\mathrm{NbO} 3) 2$ multi-self-frequencyconverter nonlinear crystals," J. Appl. Phys. 93, 3111-3113 (2003).

[10] M. O. Ramirez. D. Jaque, L. Ivleva, and L. E. Bausa, "Evaluation of ytterbium doped strontium barium niobate as a potential tunable laser crystal in the visible," J. Appl. Phys. 95, 6185-6191 (2004). 